\title{
Influence of Nonlinearity of Soil-Pile Interface on the Seismic Interaction of Nonlinear Soil-Piles-Bridge System
}

Mohanad Talal Alfach*

Faculty of Science and Engineering, School of Architecture and Built Environment, University of Wolverhampton, UK

\begin{abstract}
Post-seismic observations of recent devastating earthquakes have shown that the nonlinear behavior of the soil plays an essential and definitive role in development the damage to the system of soil-piles- structure during the earthquake. Similarly, slips or collapse the soil-piles interface may cause harmful ruptures. Consequently, an analysis of these problems is necessary to take into account the non-linearity of the soil and the possibility of a slip or collapse at the interface of soil-pile under intensive seismic loading. This study aims to investigate these interaction aspects for soil-piles-structure under real earthquake record using a global approach with a three-dimensional finite difference code - FLAC 3D (Fast Lagrangian analysis of continua in 3 dimensions). The results confirm that the non-linearity of soil and soil-pile interface has a great influence on the response of piles and structure. They show that the consideration of the plasticity of the soil leads to attenuation of efforts, especially for soft loose soils. The simulation carried out in this study illustrate that the use of weak soil-pile interface leads to an increase of bending moment accompanied by a reduction of shear and normal forces in the piles. While, for a medium or high resistance interface, the efforts induced in the piles approaching of those induced in the case of perfect contact.
\end{abstract}

Keywords: Interaction; Piles; Interface; Seismic; Plasticity; Threedimensional

\section{Introduction}

Often piles ensure the stability of structures supported by weak soils or located in seismic zones, but under strong seismic loadings, they could be probably subjected to efforts exceeds their allowable limit of seismic resistance. These efforts are particularly dangerous when the piles are anchored in nonlinear soil. The post- seismic observations and analysis have showed the fundamental role of soil-foundation-superstructure in determining the seismic damage suffered by piles and structures [17]. The analytical and numerical analyses show that the seismic piles damage is mainly due to the kinematic interaction between piles and soils or /and the inertial interaction between the superstructure and the foundation which can cause damage to the piles, particularly at the piles cap [8-12]. Subsequently, a number of analytical and numerical studies was performed on the seismic behavior of piles. These works show that the behaviour of piles under seismic loadings is a very complex problem, as it involves soil-pile interaction, piles-piles, pilecap and all piles-cap-soil and the superstructure. Due to the complexity of the nonlinear analysis of soil-piles-structure, most research in this domain was performed for elastic behaviour analysis with rigid contact between piles-soil. However, under intensive seismic loading, the nonlinear behaviour of soil is very pronounced and the rupture of the soil-piles interface is very likely. Full 3D analyses considering the effect of nonlinearity behaviour of soil- pile interface coupled with the soil nonlinear behaviour on the seismic behaviour of soil-piles-structure are very limited. Such studies were conducted in the nonlinear domain for the soil only without coupling that with the nonlinear behaviour of the soil-pile interface which is very likely to come about under strong earthquakes. The research works conducted in this study provide a thorough analysis of soil-piles-bridge interaction under seismic loads, particular attention is paid to the influence of nonlinearities behaviour of soil and soil-piles interface on the seismic response of the soil-pilesbridge system. The study is performed using a three-dimensional modelling code (FLAC 3D) [13].

This paper includes a full 3D coupled modelling of the soil-pilesuperstructure interaction under seismic loading considering the elastoplastic behaviour of the soil and soil-pile interface. The analyses provide valuable information's about the domain of validity of the linear theory. The soil behaviour is described using the non- linear MohrCoulomb criterion.

\section{Research Methodology}

\section{Soil-pile structure system and numerical model}

The model consists of an implanted group of piles in the soil. The modeling of behavior of such system under seismic loading requires specific methods to take in consideration the interaction between those different components, namely the soil-piles, pile-pile, piles-cap interaction and all piles-cap-soil with the structure. The boundaries of the model should be put sufficiently away from the structure to minimize the effect of waves reflection which leads to dense mesh. To overcome this difficulty, we use specific borders which prevent them from reflecting on the model. FLAC 3D is used in this study; this code uses the Lagrangian representation of movement. It is based on the explicit finite difference method to solve the equations of dynamic equilibrium.

\section{Flac 3D}

Since FLAC is described as an "explicit, finite difference program" that performs a "Lagrangian analysis". In the finite difference method, every derivative in the set of governing equations is replaced directly by an algebraic expression written in terms of the field variables (e.g., stress or displacement) at discrete points in space; these variables are undefined within elements (Figure 1).

\section{Lagrangian analysis}

Instead of forces, Lagrangian mechanics uses the energies in the

*Corresponding author: Mohanad Talal Alfach, Faculty of Science and Engineering, School of Architecture and Built Environment, School of Architecture and Built Environment, University of Wolverhampton, Wulfruna St, Wolverhampton WV1 1LY, UK, Tel: 01902 321000; E-mail: mohanad.alfach@wlv.ac.uk

Received June 29, 2018; Accepted August 17, 2018; Published August 22, 2018

Citation: Alfach AT (2018) Influence of Nonlinearity of Soil-Pile Interface on the Seismic Interaction of Nonlinear Soil-Piles-Bridge System. J Civil Environ Eng 8: 321. doi: 10.4172/2165-784X.1000321

Copyright: ( 2018 Alfach AT. This is an open-access article distributed under the terms of the Creative Commons Attribution License, which permits unrestricted use, distribution, and reproduction in any medium, provided the original author and source are credited. 
system. The central quantity of Lagrangian mechanics is the Lagrangian, a function which summarizes the dynamics of the entire system. Overall, the Lagrangian has units of energy, but no single expression for all physical systems. Any function which generates the correct equations of motion, in agreement with physical laws, can be taken as a Lagrangian. It is nevertheless possible to construct general expressions for large classes of applications. The non-relativistic Lagrangian for a system of particles can be defined by

$$
L \equiv T-V
$$

Where $\mathrm{T}$ is the kinetic energy, $T=m \dot{x}^{2} / 2$; and $\mathrm{V}$ is the potential energy $V=k x^{2} / 2$

So we have,

$$
\begin{aligned}
& L=\frac{1}{2} m \dot{x}^{2}-\frac{1}{2} k x^{2} \\
& \frac{d}{d t}\left(\frac{\partial L}{\partial \dot{x}}\right)=\frac{\partial L}{\partial x}
\end{aligned}
$$

If $\mathrm{T}$ or $\mathrm{V}$ or both depend explicitly on time due to time-varying constraints or external influences, the Lagrangian $\mathrm{L}(\mathrm{r} 1, \mathrm{r} 2, \ldots \mathrm{v}$, , v2, ... $\mathrm{t}$ ) is explicitly time-dependent. If neither the potential nor the kinetic energy depend on time, then the Lagrangian $\mathrm{L}(\mathrm{r} 1, \mathrm{r} 2, \ldots \mathrm{v} 1, \mathrm{v} 2, \ldots)$ is explicitly independent of time. In either case, the Lagrangian will always have implicit time-dependence through the generalized coordinates.

With these definitions Lagrange's equations of the first kind are

$$
\frac{\partial L}{\partial r_{k}}-\frac{d}{d t} \frac{\partial L}{\partial \dot{r}_{k}}+\sum_{i=1}^{c} \lambda_{i} \frac{\partial f_{i}}{\partial r_{k}}=0
$$

Where $\mathrm{k}=1,2, \ldots, \mathrm{N}$ labels the particles, there is a Lagrange multiplier $\lambda i$ for each constraint equation $f$, and

$$
\frac{\partial}{\partial r_{k}}=\left(\frac{\partial}{\partial x_{k}}, \frac{\partial}{\partial y_{k}}, \frac{\partial}{\partial z_{k}}\right), \quad \frac{\partial}{\partial \dot{r}_{k}}=\left(\frac{\partial}{\partial \dot{x}_{k}}, \frac{\partial}{\partial \dot{y}_{k}}, \frac{\partial}{\partial \dot{z}_{k}}\right)
$$

The Euler-Lagrange equations, or Lagrange's equations of the second kind

$$
\frac{d}{d t}\left(\frac{\partial L}{\partial \dot{q}_{j}}\right)=\frac{\partial L}{\partial q_{j}}
$$

\section{Reference example: Elastic}

The reference example consists from a group of $(2 \times 3)$ floating

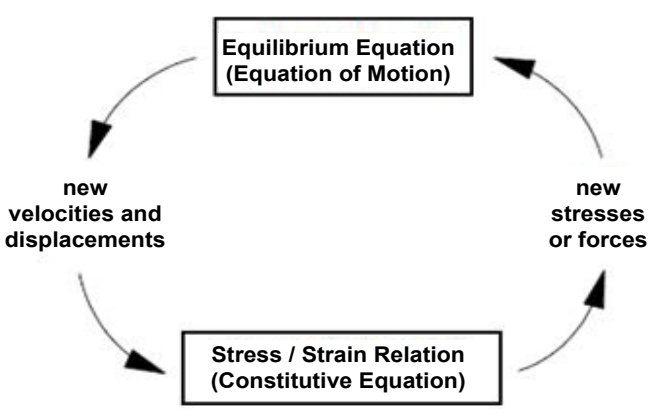

Figure 1: Basic explicit calculation cycle. piles with length $\left(L_{p}=10.5 \mathrm{~m}\right)$. The group is implanted into a layer of homogeneous soil with a depth of $(15 \mathrm{~m})$ and embedded in a cap of $(1 \mathrm{~m})$ thick (Figure 2). The characteristics of soil, piles, and superstructure are given in Tables 1 and 2. The mechanical and geometrical characteristics of reference example are plotted in Figure 2a. The pile's heads $\left(D_{p}=80\right.$ $\mathrm{cm})$ are embedded in cap of thick $\left(\mathrm{e}_{\mathrm{c}}=1 \mathrm{~m}\right)$ with rigid contact, the spacing between piles is $\left(\mathrm{S}=3.75 \mathrm{D}_{\mathrm{p}}=3 \mathrm{~m}\right)$. To avoid the complexity of soil-cap interaction, the cap was placed in $(0.5 \mathrm{~m})$ above the soil. The piles were modeled as embedded structural pile (2D) elements with perfect contact with the soil in this reference example. In this reference example, the behavior of soil-pile-structure is assumed to be elastic with Rayleigh damping for the soil, the factor of damping used is (5\%) for the soil and (2\%) for the structure. The equations of Rayleigh damping are expressed in damping matrix form $\mathrm{C}$ with components proportional to the mass $(\mathrm{M})$ and stiffness $(\mathrm{K})$ matrices:

$$
C=\alpha M+\beta K
$$

Where $\alpha$ the mass-proportional damping constant; and $\beta$ the stiffness-proportional damping constant.

The Shear modulus of the soil $\mathrm{G}=2.76 \mathrm{MPa}$, while, the bulk modulus of the soil $\mathrm{K}=26.66 \mathrm{MPa}$, which have calculated by using the following equations:

$$
\begin{aligned}
& G=\frac{E}{2(1+\mu)} \\
& K=\frac{E}{3(1-2 v)}
\end{aligned}
$$

Where E Young modulus in $\left(\mathrm{N} / \mathrm{m}^{2}\right)$; and $\mu$ Poisson ratio.

The fundamental frequency of soil is $(0.67 \mathrm{HZ})$ which was calculated in two ways: firstly by using the following equation for $\mathrm{V}_{\mathrm{s}}=40 \mathrm{~m} / \mathrm{sec}$ in the first mode of vibration, secondly the value has been checked by frequency analysis of free field soil by using Flac 3D.

$$
T_{n}=\frac{4 H}{(2 n-1) V_{s}}
$$

Where $T_{n}$, The fundamental period of the soil in (sec);

$\mathrm{H}$, The depth of the soil under the structure in $(\mathrm{m})$;

$\mathrm{V}_{\mathrm{s}}$, Shear velocity in the soil in $(\mathrm{m} / \mathrm{sec})$; and $\mathrm{n}$ Mode number.

The superstructure is modeled by a column which supports mass in its head ( $M=350$ Tons). The rigidity of the superstructure and its frequency (assumed fixed at the base) are equals to $\mathrm{K}_{\mathrm{st}}=86840 \mathrm{KN} / \mathrm{m}$ and $\mathrm{F}_{\mathrm{st}}=2.5 \mathrm{HZ}$. They were determined by the following expressions:

$$
\begin{aligned}
& k_{s t}=\frac{3\left(E_{s t} \cdot I_{s t}\right)}{H_{s t}^{3}} \\
& F_{s t}=1 / 2 \pi \sqrt{\frac{K_{s t}}{m_{s t}}}
\end{aligned}
$$

Where $\mathrm{E}_{\mathrm{st}}$, Young modulus of the superstructure in $\left(\mathrm{N} / \mathrm{m}^{2}\right)$;

$\mathrm{I}_{\mathrm{st}}$, Inertia moment of the Super structure in $\left(\mathrm{m}^{3}\right)$;

$\mathrm{K}_{\mathrm{st}}$, Bulk modulus of the superstructure in $(\mathrm{N} / \mathrm{m})$;

$\mathrm{m}_{\mathrm{st}}$, Mass of the superstructure in $(\mathrm{Kg})$; 

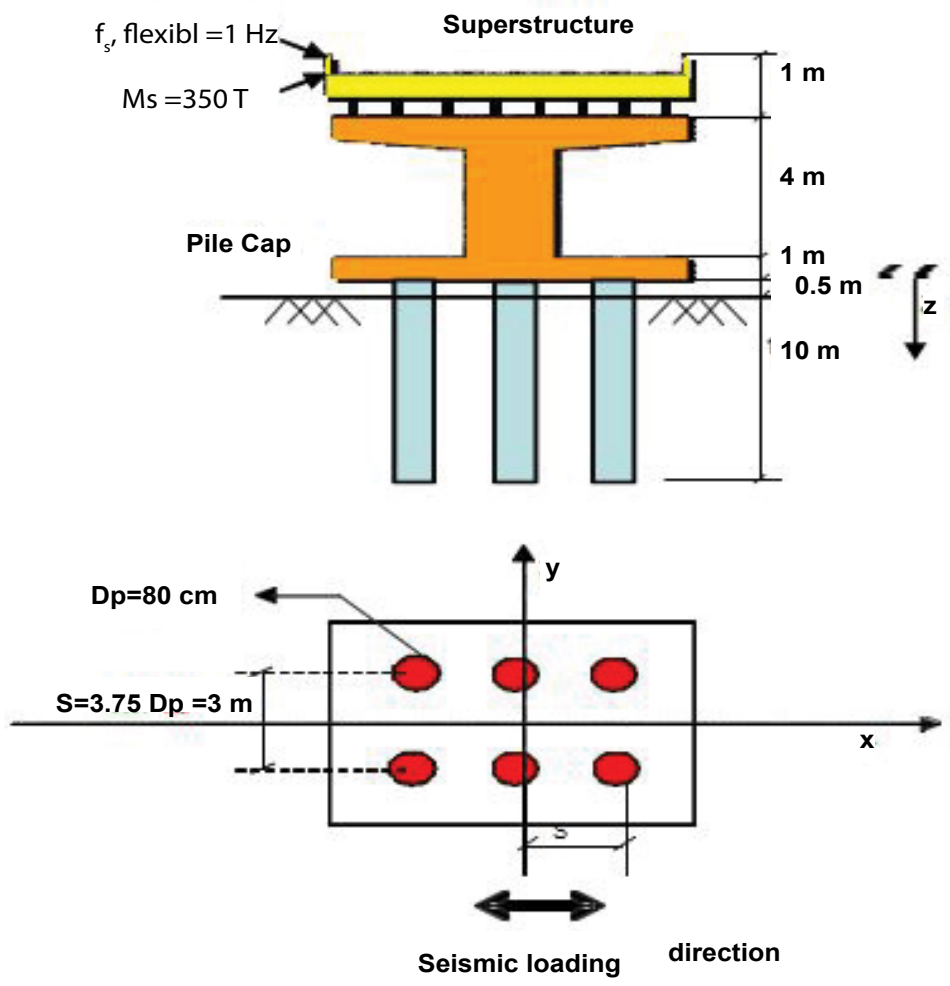

Figure 2a: Problem under consideration (System geometry).

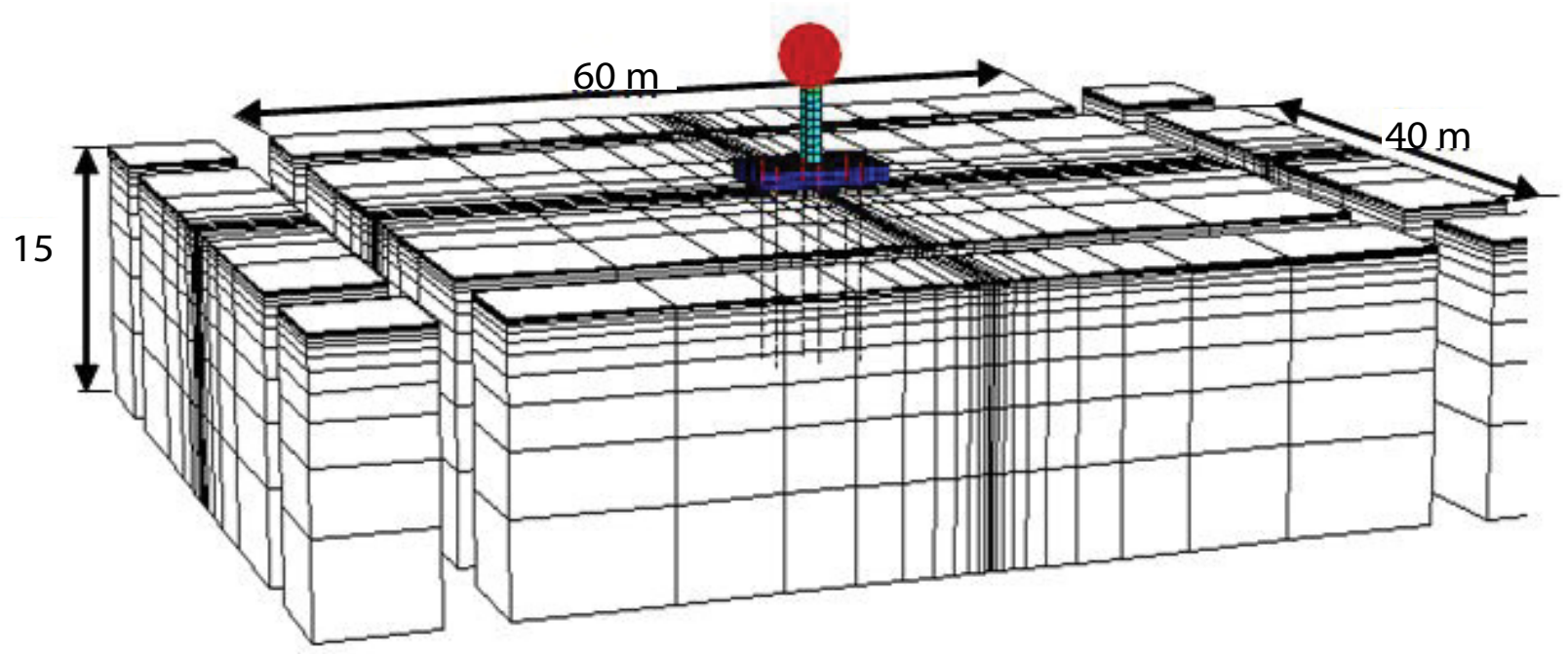

Figure 2b: Problem under consideration (System geometry).

$\mathrm{H}_{\mathrm{st}}$, Height of the superstructure in (m).

The frequency of the superstructure taking in consideration the soil-structure interaction is $\mathrm{f}_{\text {st,flex }}=1.1 \mathrm{~Hz}$ (including SSI), which was calculated by frequency analysis of the global problem of soil-structure interaction in Flac 3D.

$\rho, \mathrm{E}$ and $v$ are the density, young modulus and the coefficient of Poisson. $\xi$ is the factor of damping. Dp is the pile diameter. $\mathrm{E}^{*} \mathrm{~A}$ and $E^{*} \mathrm{I}$ : are the axial and flexural rigidity, noting $\mathrm{E}^{*} \mathrm{~A}=1005.3 \mathrm{e}^{6} \mathrm{~N} / \mathrm{m}$ and $\mathrm{E}^{*} \mathrm{I}=40.2 \mathrm{e}^{6} \mathrm{~N} \cdot \mathrm{m}^{2}$ for the reference example. The used mesh shown in Figure 2b includes (3856) zones of (8) nodes and (138) threedimensional beams of 2 nodes. The mesh was refined around the piles and near the superstructure where inertial forces induce high stresses.

\section{Real seismic loading record}

The seismic loading chosen in this research is the one recorded in Kocaeli at Turkey on 17/08/1999 (Station AMBARLI; KOERI source). This loading which is applied in (X) direction as a speed at the base of the soil is shown in Figure 3. The maximum amplitude of this loading is $(40 \mathrm{~cm} / \mathrm{s})($ acceleration maximum $=0.247 \mathrm{~g})$. 

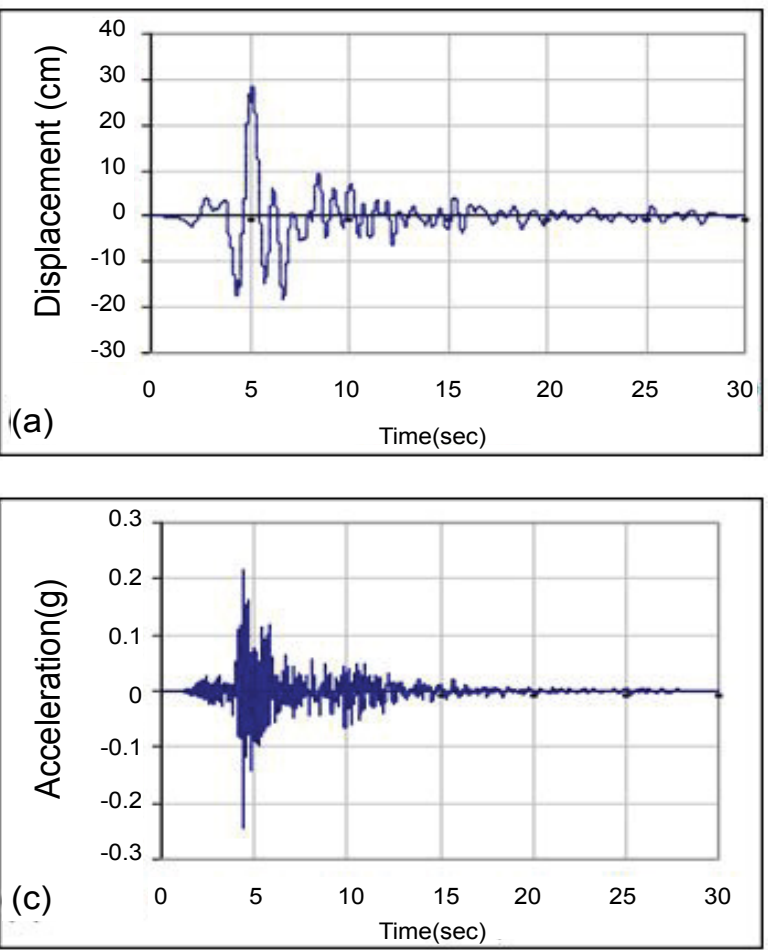
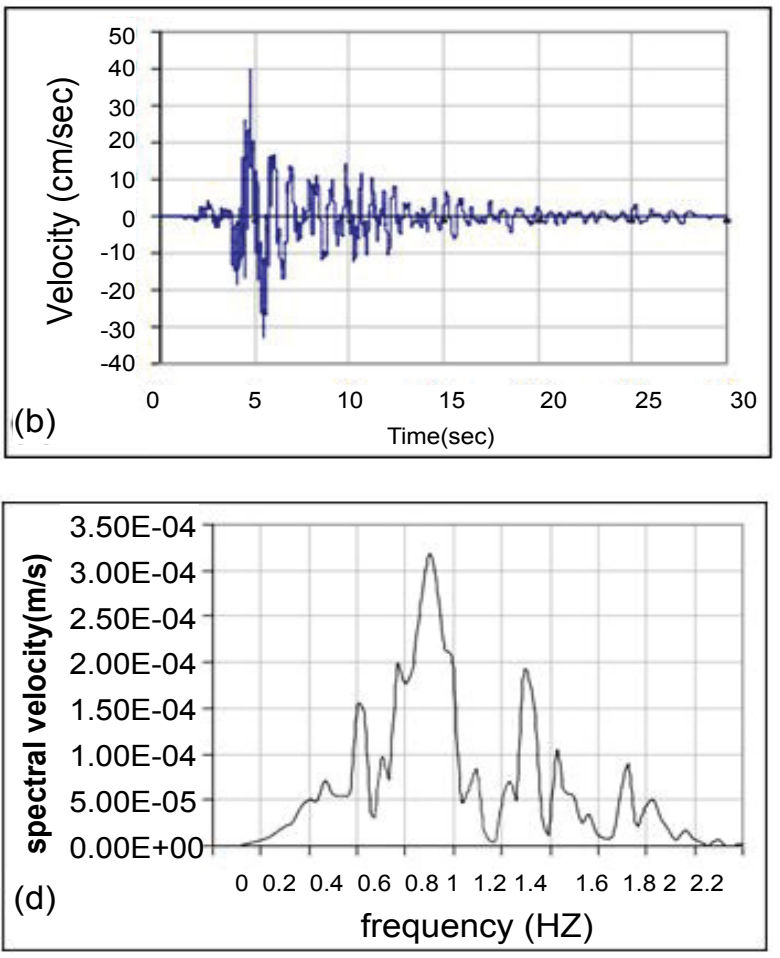

Figure 3: Kocaeli earthquake record (1999) - a) Displacement, b) Velocity, c) Acceleration, d) Fourier spectra of Velocity component.

The spectrum of Fourier corresponding to the used seismic loading illustrated in Figure 3. We note that the frequencies involved are less than (3) $\mathrm{Hz}$ with the maximum peak for $(\mathrm{F}=0.9 \mathrm{~Hz})$ which is between the fundamental frequency of the soil $\left(\mathrm{F}_{1}=0.67 \mathrm{~Hz}\right)$ and the frequency of structure $(\mathrm{Fss}=1.1 \mathrm{~Hz})$, Hence, the choice of this loading in our analysis. Also, note that a first peak is observed for frequency ( $\mathrm{F}=0.6$ $\mathrm{Hz}$ ) which is very close to the fundamental frequency of soil.

Table 3 shows the efforts induced in the piles under Kocaeli earthquake loading. Since we have interaction between the three components of the structural system (superstructure, cap, and the piles), the internal forces induced at the base of the superstructure affect the internal forces induced in the piles, so we can't compare correctly the internal forces induced in the piles without normalized these efforts to the forces induced at the base of the superstructure. Hence, in order to compare the obtained results, induced efforts are normalized to inertial forces of the superstructure as follows:

$$
\begin{aligned}
T^{*} & =\frac{T}{T_{c a p}} \\
M^{*} & =\frac{M}{m_{s t} a_{s t} H_{s t}}
\end{aligned}
$$

Where:

$\mathrm{m}_{\mathrm{st}}$ The bending moment at the base of the superstructure;

$\mathrm{T}_{\text {cap }}$ and $\mathrm{a}_{\text {st }}$ denote the inertial force induced at the cap and the acceleration of the superstructure mass; and $\mathrm{H}_{\text {st }}$ Superstructure height.
Influence of soil-pile interface on the seismic response of the soil-piles-structure system

When we take in consideration the interaction of solids in contact with deformability characteristics very different such as soil and concrete, rupture is often likely to occur at the interface. Actually, the contact between soil-pile is not perfect which leads very likely to rupture at the soil-pile interface under intensive seismicloading.

Presentation of the soil-pile interface: The soil-pile interface is modeled by spring and dashpot. The behavior of this interface is described by its rigidity $\mathrm{K}$ and the parameters of its resistance $\mathrm{C}_{\mathrm{s}}$ and $\varphi_{\mathrm{s}}$ (Figure 4). We assume an elastic behavior in the normal direction of this interface by introducing a resistance in this direction. On the other side, a possible rupture in this interface occurs when the shear stress reaches its limit. The rigidity of the interface taken equal to shear modulus of adjacent soil. The inelastic interface logic works in the following waY:

The Coulomb shear-strength criterion limits the shear force by the following relation:

$F s_{\text {max }} c A+\tan \varphi(F n-p A)$

where $\mathrm{c}$ is the cohesion along the interface;

$\varphi$ is the friction angle (degrees) of the interface surface;

and $\mathrm{p}$ is pore pressure (interpolated from the target face),

If the criterion is satisfied (if $|\mathrm{Fs}| \geq \mathrm{Fs}_{\max }$ ), then sliding is assumed to occur, and $|\mathrm{Fs}|=\mathrm{Fs}_{\max }$, with the direction of shear force preserved. During sliding, shear displacement may cause an increase in the effective normal stress on the joint, according to the relation.

$$
\sigma_{n}=\sigma_{n}+\frac{\left|F_{s}\right|_{o}-F_{s \max }}{A k_{s}} \tan \psi k_{n}
$$



made.

where $\psi$ is the dilation angle [degrees] of the interface surface; and

$\left|\mathrm{F}_{\mathrm{s}}\right|_{\mathrm{o}}$ is the magnitude of shear force before the above correction is

The shear behavior of the pile, during relative shear displacement between the pile/soil interface, as shown in Figure 4, is described numerically by: (1) The soil shear stiffness $\mathrm{k}_{\mathrm{s}}$; (2) The soil cohesive strength $c_{S} ;(3)$ The soil friction angle $\varphi_{S} ;(4)$ The soil exposed perimeter $\mathrm{p}_{\mathrm{s}}$; and (5) The effective confining stress $\sigma_{\mathrm{m}}$

The effective confining stress $\sigma_{\mathrm{m}}$ acts in the plane perpendicular to the pile axis, and is computed at each nodal point along the pile axis, based on the stress acting in the zone to which the nodal point is linked. Denote the pile-axis direction as $\mathrm{x}$, and denote the principal stresses acting in the yz plane as $\sigma_{1}$ and $\sigma_{2}$, such that $\sigma_{1}>\sigma_{2}$ (tension positive). Then the value of $\sigma_{\mathrm{m}}$ is taken as

$$
\sigma_{m}=-\left(\frac{\sigma_{1}+\sigma_{2}}{2}+p\right)
$$

Where $\psi$ is the dilation angle [degrees] of the interface surface; and made.

$\left|\mathrm{F}_{\mathrm{s}}\right|_{\mathrm{o}}$ is the magnitude of shear force before the above correction is

\section{Case of frictional soil}

In order to study the nonlinear behavior of frictional soil under seismic loading, the simulation analyses in this section have carried out for frictional soil $\left(\mathrm{C}=2 \mathrm{KPa}, \varphi=30^{\circ}, \psi=20^{\circ}\right)$ firstly in case of perfect soil-pile contact, then, coupled with elastic and nonlinear behavior interfaces. Cohesive interfaces with several values for the resistance of the soil-pile vary from moderate to relatively strong interfaces $\mathrm{C}_{\mathrm{s}}=50,100,150 \mathrm{KPa}$ were examined. The responses of the piles in case of using nonlinear interface were compared with responses to an interface infinitely resistant (perfect contact). The applied seismic loading is the real record of Turkey [14].

\section{Results and Discussion}

The results of the seismic analyses of soil-pile-bridge system in cases of using elastic and non-linear behavior for the soil-pile interface are given in Table 4 and Figures 5, 6 and 7. It is noted that the non-linearity of the interface leads to a sharp decrease of the internal force in case of using weak interface $(\mathrm{C}=50 \mathrm{KPa})$, while, these induced forces increase gradually for $(\mathrm{C}=100-150 \mathrm{KPa})$ and become for $(\mathrm{C}=150 \mathrm{KPa})$ very close of the induced forces in case of using elastic interface. Moreover, pay regard to the interface conduct to decrease the amplification of the structure. This pronounced decrease for poor interface characteristics $(50 \mathrm{KPa})$, was accompanied with an amplification of lateral acceleration (35\%) less than that obtained for perfect contact between soil and piles. This trend is confirmed for internal forces in piles. The profiles of shear force and bending moment (Figure 5) show that the responses for rigidities of the interface ( 100 and $150 \mathrm{KPa}$ ), are very close to those obtained for perfect contact. For weak resistance interface $\left(\mathrm{C}_{\mathrm{s}}=50 \mathrm{KPa}\right)$, the profiles of the bending moment and shear force are completely changed.

We observe reduction of maximum shear force at pile head in accordance with that of the lateral acceleration. However, the bending moment increases significantly. The value of the maximum bending moment normalized obtained for $\left(\mathrm{C}_{\mathrm{s}}=50 \mathrm{KPa}\right), \mathrm{M}^{*}=0.223$ is 4 times higher than that obtained for an elastic interface which can be attributed to the sliding of the interface or rather separation soil-piles reflected in the relative displacements between the pile and surrounding soil, this significant increase in bending moment indicates a reduction in the rigidity of soil-piles and leads to high deformability in piles. As for the normal force, we note higher sensitivity depending on the resistance of the interface. The decreasing of interface resistance translates into a decrease of normal force in piles. For a strong resistance of the interface $\left(\mathrm{C}_{\mathrm{s}}=150 \mathrm{KPa}\right)$, the response obtained is very close to that obtained for perfect contact. For medium resistance $\left(\mathrm{C}_{\mathrm{s}}=100 \mathrm{KPa}\right)$, we obtain $\mathrm{s}$ a drop in maximum normal force of about $(22 \%)$. For weak resistance $\left(\mathrm{C}_{\mathrm{s}}=50 \mathrm{KPa}\right)$, the profile of normal force is strongly effected with a very sharp decline of over (85\%). This is due to slippage at the soil/pile interface. Finally, the consideration of a soil/pile interface is essential in case of weak resistance. In case of strong resistance of the interface, taking account soil plasticity is sufficiently, and the soil/pile interface can be modeled by a perfect contact.

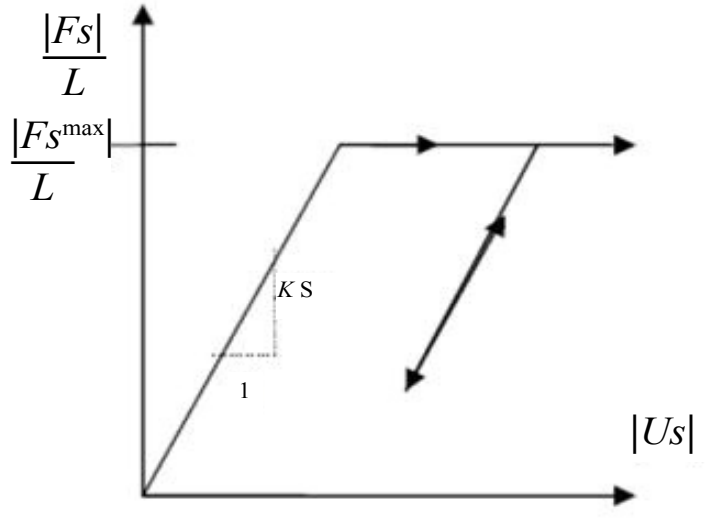

Friction unit according to the relative tangential displacement soil-pile

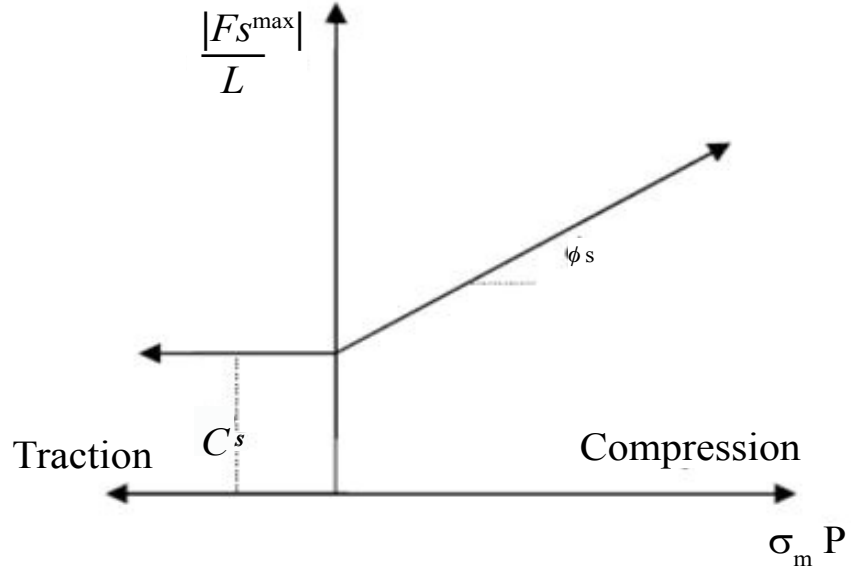

criterion of failure of tangential interface

(b)

a)

Figure 4: Behavior of the soil-pile interface in the tangential direction (Flac 3D Manual). 


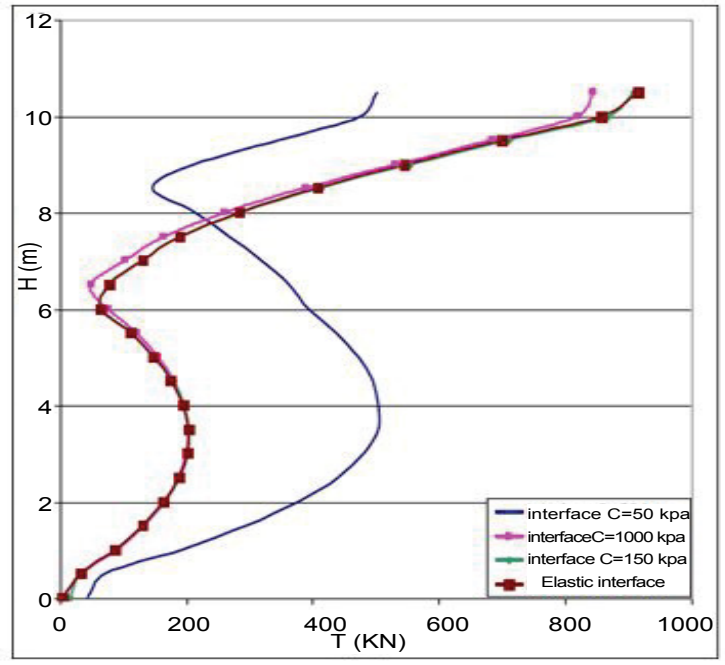

a) Maximum Shear Force

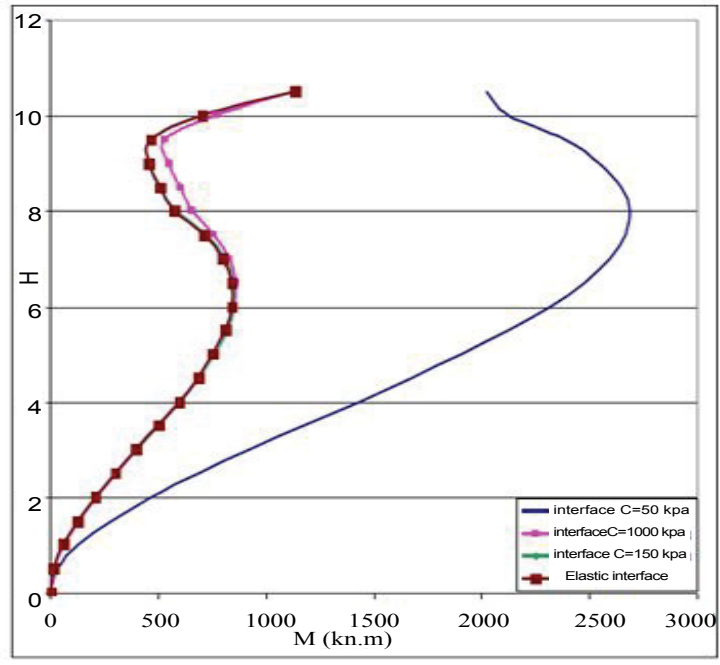

b) Maximum Bending Moment

Figure 5: Influence of soil-pile interface on the dynamic forces in the corner piles (Frictional soil, Earthquake of Turkey, $\mathrm{V}_{\mathrm{g}}=40 \mathrm{~cm} / \mathrm{s}$ )

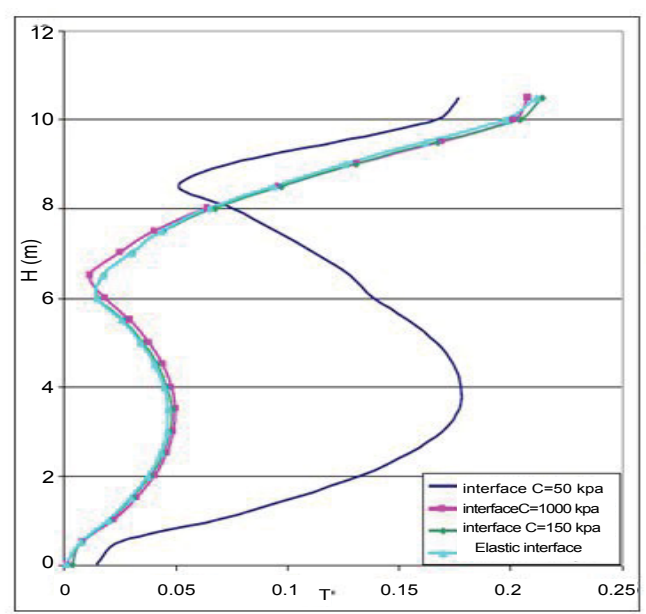

a) Maximum normalized Shear Force

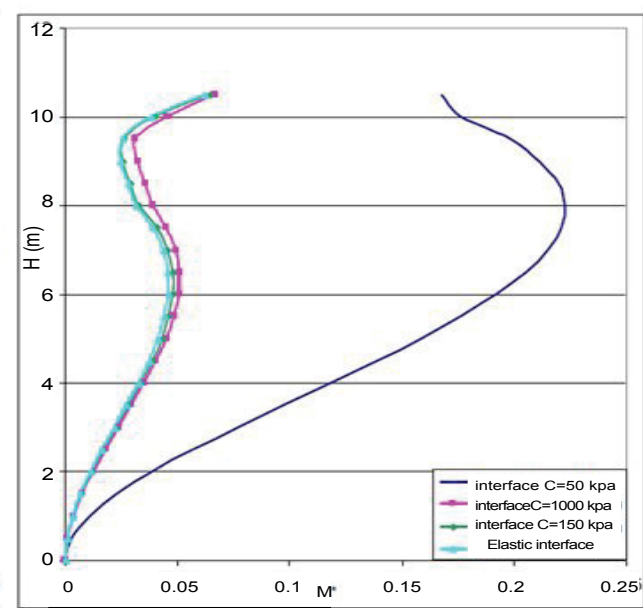

b) Maximum normalized BendingForce

Figure 6: Influence of soil-pile interface on the normalized dynamic forces in the corner piles (Frictional soil, Earthquake of Turkey, $\mathrm{V}_{\mathrm{g}}=40 \mathrm{~cm} / \mathrm{s}$ ).

\begin{tabular}{|c|c|c|c|c|c|c|}
\hline Material & Diameter (m) & Mass Density $(\rho)\left(\mathrm{kg} / \mathrm{m}^{3}\right)$ & Young Modulus E (Mpa) & Poisson ratio (u) & Damping ratio $\xi(\%)$ & Height (m) \\
\hline Pile & 0.8 & 2500 & 420000 & 0.3 & 2 & 10 \\
\hline Soil & & 1700 & 8 & 0.45 & 5 & 15 \\
\hline
\end{tabular}

Table 1: Elastic property of the soil and piles materials.

\begin{tabular}{|c|c|c|c|c|}
\hline $\mathbf{( k g / \mathbf { m } ^ { 3 } )}$ & $\mathbf{E}_{\mathbf{s t}}(\mathbf{M p a})$ & $\mathbf{u}_{\mathbf{s t}}$ & $\boldsymbol{\xi}(\%)$ & Mass (Ton) \\
\hline 2500 & 80000 & 0.3 & 2 & 350 \\
\hline
\end{tabular}

Table 2: Elastic property of the super-structure.

\begin{tabular}{|c|c|c|c|c|c|c|c|c|}
\hline \multirow{3}{*}{ Seismic Loading } & \multirow{3}{*}{ ast $\left(\mathrm{m} / \mathrm{s}^{2}\right)$} & \multirow{3}{*}{${ }^{\mathrm{a}} \mathrm{Cap}\left(\mathrm{m} / \mathrm{s}^{2}\right)$} & \multicolumn{4}{|c|}{ Internal forces } & \multirow{2}{*}{\multicolumn{2}{|c|}{$\begin{array}{c}\text { Normalized forces } \\
\text { Corner piles }\end{array}$}} \\
\hline & & & \multicolumn{2}{|c|}{ Central piles } & \multicolumn{2}{|c|}{ Corner Piles } & & \\
\hline & & & $\mathrm{T}_{\max }(\mathbf{k N})$ & $M_{\max }(k N . m)$ & $\mathbf{T}_{\max }(\mathbf{k N})$ & $M_{\max }(k N . m)$ & $\mathbf{T}_{\max }^{*}$ & $\mathbf{M}_{\max }^{*}$ \\
\hline Turkey & 11.28 & 8.385 & 675.8 & 954.4 & 1016.1 & 1099 & 0.196 & 0.05 \\
\hline
\end{tabular}

Table 3: Reference example: Response of a group of $(2 \times 3)$ piles for turkey loading. 


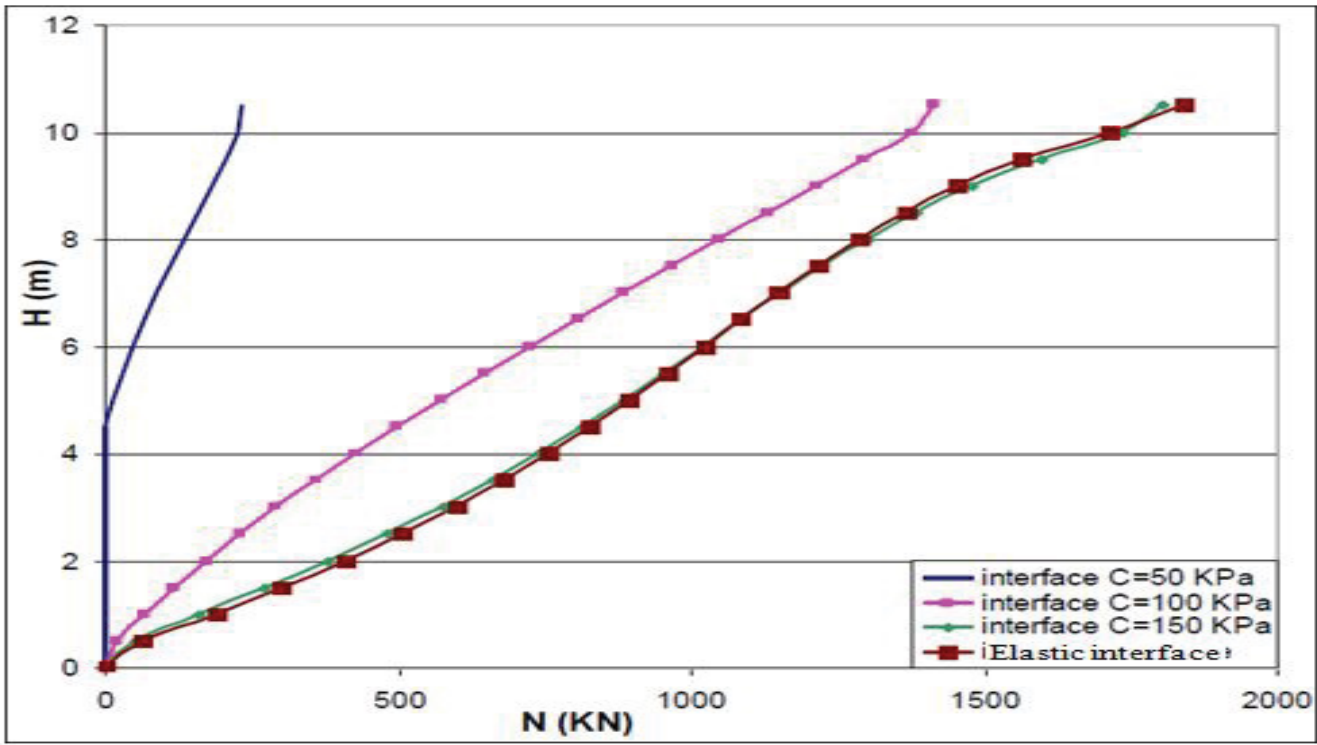

Figure 7: Influence of soil-pile interface on the normal force in the corner pile (Frictional soil, Earthquake of Turkey, $\mathrm{V}_{\mathrm{g}}=40 \mathrm{~cm} / \mathrm{s}$ ).

\begin{tabular}{|c|c|c|c|c|c|c|c|}
\hline \multirow{3}{*}{ Interface ( $\mathrm{kPa})$} & \multirow{3}{*}{$A_{c c} \operatorname{mass}\left(m / s^{2}\right)$} & \multirow{3}{*}{$A_{c c} \operatorname{Cap}\left(m / s^{2}\right)$} & \multirow{2}{*}{\multicolumn{3}{|c|}{$\begin{array}{c}\text { Dynamic forces } \\
\text { Corner Piles }\end{array}$}} & \multirow{2}{*}{\multicolumn{2}{|c|}{$\begin{array}{c}\text { Normalized forces } \\
\text { Corner piles }\end{array}$}} \\
\hline & & & & & & & \\
\hline & & & $N_{\max }(k N)$ & $\mathrm{T}_{\max }(\mathrm{kN})$ & $M_{\max }$ (kN.m) & $\mathbf{T}_{\max }^{*}$ & $\mathbf{M}_{\max }^{*}$ \\
\hline Elastic & 9.567 & 6.592 & 1840.2 & 917.3 & 1140 & 0.2 & 0.062 \\
\hline 50 & 6.304 & 4.19 & 235.3 & 504.8 & 2690 & 0.178 & 0.223 \\
\hline 100 & 8.81 & 6.585 & 1413.2 & 844.7 & 1132 & 0.2 & 0.067 \\
\hline 150 & 9.27 & 6.718 & 1804.2 & 909.1 & 1145 & 0.21 & 0.064 \\
\hline
\end{tabular}

Table 4: Influence of soil-pile interface (Frictional soil, Earthquake of Turkey, $V_{g}=40 \mathrm{~cm} / \mathrm{s}$ ).

\section{Conclusion}

This study was devoted to comprehensive numerical modeling of soil-pile-bridge interaction under seismic loading. Attention particular has been paid to the influence of nonlinearities of soil and soil-pile interface on the seismic response. The research in this study was conducted using a three-dimensional modeling by finite difference program (FLAC 3D). The plasticity of soil influence significantly the seismic response of the system for cohesive soil, the plasticity propagating from the base of this massive which damped the transmission of energy to the surface and the superstructure. Taking into account the plasticity of the soil leads to attenuation of efforts, especially for soft loose soils. The introduction of specific elements of the soil-pile interface becomes possible grace the advances in numerical calculation methods. The simulations performed show that for a poor resistance of soil-piles interface, the use of interface elements leads to an increase of bending moment accompanied by a decline of shear and normal forces in the piles. For a medium or high resistance interface, the efforts in the piles approaching of that in the case of perfect contact, in this case, the taking into account the soil plasticity is sufficient and the use of a more expensive model with interface elements is no longer justified.

\section{References}

1. Mizuno H, liba M, Kitagawa $Y$ (1982) Shaking table testing of seismic building pile-two layered soil interaction. Proceedings of the 8th World Conference on Earthquake Engineering 3: 649-656.
2. Gazetas G, Fan K, Kaynia A, Kausel E (1991) Dynamic interaction factors for floating pile groups. J Geotech Eng-ASCE 117: 1531-1548.

3. Tao X, Kagawa T, Minowa C, Abe A (1998) Verification of dynamic soil-pile interaction. In: Geotechnical Earthquake Engineering and Soil Dynamics. ASCE pp: 1199-1210.

4. Boulanger R, Curras C, Kutter J, Wilson D, Abghari A (1999) Seismic soil-pilestructure interaction experiments and analyses. J Geotech Geoenviron 125 750-759.

5. Miura F (2002) Some typical examples of damage to pile foundation by the 1995 Hoygoken Nambu earthquake International workshop on micropiles-IWM 2002 Session VI, detailed seismic design and performance issues, Venice, Italy.

6. Tuladhar R, Maki T, Mutsuyoshi H (2007) Cyclic behavior of laterally loaded concrete piles embedded into cohesive soil. Earthq Eng Struct Dyn 37: 43-59.

7. Sushma P, Pradeep Kumar R (2012) Numerical modeling of interface between soil and pile to account for loss of contact during seismic excitation. 15 WCEE, Lisbon.

8. Gazetas G, Mylonakis G (1998) Seismic soil-structure interaction: New evidence and emerging issues. Emerging issues paper. Geotechnical Earthquake Engineering and Soil Dynamics Geo-Institute: ASCE Conference New Mexico.

9. Nikolaou S, Mylonakis G, Gazetas G, Tazoh T (2001) Kinematic pile bending during earthquakes: analysis and field measurements. Géotechnique 51: 425-440.

10. Maheshwari BK, Truman KZ, El-Naggar MH, Gould PL (2004) Threedimensional nonlinear analysis for seismic soil- pile-structure interaction. Soil Dyn Earthq Eng 24: 343-356.

11. Finn WDL (2005) A study of piles during earthquakes: Issues of design and analysis. B Earth Eng 3: 141-234

12. Gerolymos N, Escoffier S, Gazetas G, Garnier J (2009) Numerical modeling of centrifuge cyclic lateral pile load experiments. Earthq Eng Eng Vib 8: 61-76. 
Citation: Alfach AT (2018) Influence of Nonlinearity of Soil-Pile Interface on the Seismic Interaction of Nonlinear Soil-Piles-Bridge System. J Civil Environ Eng 8: 321. doi: 10.4172/2165-784X.1000321

13. FLAC: Fast Lagrangian Analysis of Continua (2005) Verification problems and example applications. Vol. I. User's Manual, Vol. II (2nd edn) (FLAC3D Version 3.0), Minneapolis, Minnesota, USA.
14. Tramutoli V, Cuomo V, Filizzola C, Pergola N, Pietrapertosa C (2005) Assessing the potential of thermal infrared satellite surveys for monitoring seismically active areas: The case of Kocaeli (Izmit) earthquake, August 17, 1999. Remote Sens Environ 96: 409-426. 